\title{
Displacement uncertainties with multiview correlation schemes
}

François Hild ${ }^{1}$ and Stéphane Roux ${ }^{1}$

\begin{abstract}
Multiview correlation is based on the use of explicitly parameterizing surfaces whose deformation is to be measured. In the present analyses, the surfaces are modeled as triangular facets (for local approaches), and with finite element meshes (for global approaches) using a linear interpolation between nodal displacements. Displacement uncertainty due to the presence of noise in the images is considered. The noise floor levels of the latter are compared for both local and global cases with two different sets of pictures. A priori estimates based upon the Hessian of the registration procedure are derived analytically, and a simplified expression is provided that is shown to be in very good agreement with their a posteriori evaluations. The uncertainty difference between local and global approaches is interpreted from their different spatial resolutions.
\end{abstract}

\section{Keywords}

Covariance matrix; Noise floor level; Stereocorrelation; Uncertainty quantification.

\footnotetext{
1 Université Paris-Saclay, ENS Paris-Saclay, CNRS

LMT-Laboratoire de Mécanique et Technologie, Cachan, France
} 


\section{Introduction}

StereoDIC (also known as 3D-DIC or stereocorrelation) is a well-established measurement technique $[1,2]$. As a result, commercial systems are currently available (e.g., $[3,4,5,6,7,8])$. The standard way of addressing shape and deformation measurements was to extend matching concepts (i.e., based upon local registrations) originally developed for 2D-DIC to the analysis of the deformation of $3 \mathrm{D}$ surfaces $[9,10,11,12,13]$. As a result, the output of virtually all analyses are clouds of $3 \mathrm{D}$ points. Consequently, the comparison of measurements with the theoretical surface definitions (e.g., CAD or FE parameterizations) requires post-processing steps. The most common methods rely on Iterative Closest Point (ICP) algorithms [14, 15] and may induce additional errors or uncertainties in the determination of deviations between theoretical and actual surfaces [16].

To overcome some of the previous issues, global approaches have been introduced, in which the prior knowledge of the surface of interest is explicitly accounted for $[17,5]$. The calibration step enables the camera frame to be repositioned within that of the numerical (i.e., virtual) calibration target or the surface of interest if self-calibration is performed [18]. Up to now, two different discretizations of the reference object have been considered, namely, non-uniform rational B-splines (or NURBS) $[18,19,20]$ and finite-element based descriptions using triangular [21,22] or quadrangular [23] meshes.

Any vision-based technique requires uncertainty quantifications to be performed so that they be utilized as measurement tools. First, uncertainties were evaluated by propagating acquisition noise for the determination of

\section{Corresponding author:}

François Hild. Email: francois.hild@ens-paris-saclay.fr 
calibration parameters and 3D positions. Such derivations correspond to the socalled Rao and Cramér lower bounds [24, 25]. The theoretical derivations were validated against simulations [26] and actual experimental configurations [27]. Synthetic analyses were subsequently performed via Monte-Carlo simulations to assess the sensitivities to various calibration parameters [28, 29]. Lighting variations were added as a source of error, and artificial deformations were studied as well [30]. Such frameworks enable simulators to fully characterize the uncertainties and propose guidelines for good practices [31]. This bibliographic review shows that displacement uncertainties received less attention than the other studied quantities (including systematic errors). The present study will specifically deal with displacements, and it will be assumed that the only source of deviation between measured and actual displacements is due to the matching algorithm and its sensitivity to acquisition noise.

The paper is organized as follows. First, the framework of multiview correlation (which is a generalization of stereo-correlation to an arbitrary number of cameras) will be recalled when applied to displacement field measurements. It will enable for the derivation of the covariance matrix of the complete set of measured degrees of freedom. Analyses of two artificial cases are then performed to compare local and global stereocorrelation based on triangular tessellations. In particular, the a priori uncertainty estimates based on the global Hessian will be compared to a posteriori evaluations. Last, a compact expression is proposed to estimate a priori the uncertainty due to noise, which accounts for the effect of the different parameters of image acquisitions.

\section{Local / global multiview correlation}

In the following, the general framework of multiview correlation procedures [20] is followed in order to measure displacement fields and assess their uncertainties due to image noise (i.e., so-called noise floor levels [32]). 
Generally, there are three steps in the use of stereovision systems for shape and deformation measurements, namely, i) calibration of the stereo-setup, ii) shape and iii) subsequent displacement field measurements [1]. Multiview procedures follow the same steps [20]. In the sequel, only the third step (i.e., displacement field measurements) will be analyzed in details, although the first two steps can be studied in the same way.

The starting point is the mathematical model of the surface of interest and its deformation [17], which is not taken into account in standard stereoDIC procedures [1]. The parameterization of the sought displacement field $\mathbf{U}$ is written as

$$
\mathbf{U}(\mathbf{X})=\sum_{i=1}^{n_{p}} \sum_{j=1}^{3} U_{i j} N_{i}(\mathbf{X}) \mathbf{e}_{j}
$$

with respect to the initial shape of the surface of interest, where $\mathbf{X}$ denotes the vector defining the position of any point on the $3 \mathrm{D}$ surface, $\mathbf{U}_{i}=\sum_{j=1}^{3} U_{i j} \mathbf{e}_{j}$ the displacement of the $i$-th (out of $n_{p}$ ) remarkable point (e.g., control point of NURBS-based descriptions, mesh node for finite element formulations), $N_{i}(\mathbf{X})$ the corresponding (scalar) interpolation function (e.g., blending function in NURBS-based descriptions, finite element shape functions), and $\mathbf{e}_{j}$ the unit vectors (i.e., $j=1,2,3$ ) of the reference frame.

Images as captured by different cameras are corrupted by random noise. The latter can only be characterized statistically. It is assumed in the present study that this noise is Gaussian, white (i.e., with no spatial correlation from pixel to pixel) and uniform (i.e., noise variance is considered constant for each camera). Because of this noise, the measured kinematic degrees of freedom $U_{k}$ (where the index $k$ corresponds to the vectorization of $U_{i j}$ with respect to the indices $i$ and $j$ ) are corrupted by a noise $\eta$, which will lead to displacement fluctuations $\delta U_{k}$ whose statistical properties quantify the displacement uncertainties. In particular, because image noise is assumed to be small in amplitude, the 
model relating images to kinematic parameters can be linearized about the converged solution $[33,34,24,25]$, and hence displacement uncertainties will inherit from image noise a Gaussian distribution (because of the stability of Gaussian random variables under linear transforms). For the same reasons, the expected value of displacement fluctuation $\left\langle\delta U_{k}\right\rangle=0$ is null, and hence the kinematic measurements are unbiased. The entire displacement noise statistics is fully determined by its covariance matrix [35], $\operatorname{Cov}_{k m}=\left\langle\delta U_{k} \delta U_{m}\right\rangle_{\eta}$ (where $\langle\cdot\rangle_{\eta}$ denotes the expected value over noise), which is the aim of the present study.

Let us consider a set of $n_{c} \geq 2$ cameras. Each of them is defined by its projection matrix $\left[\mathbf{M}^{c}\right]$ that allows the homogeneous coordinates of $3 \mathrm{D}$ points $\{\mathbf{X}\}$ to be related to those of the pixel positions $\left\{\mathbf{x}^{c}\right\}$ in camera $c$ plane by

$$
s^{c}\left\{\mathbf{x}^{c}\right\}=\left[\mathbf{M}^{c}\right]\{\mathbf{X}\}
$$

where $s^{c}$ is the local scale factor.

It is convenient to decompose the surface of interest $\mathcal{S}$ into $n_{s}$ different subsurfaces, $\mathcal{S}_{j}$, and to introduce a weight $\omega_{j}^{c}$ equal to 1 when the surface is visible, and 0 when it is hidden, for camera $c=1, \ldots, n_{c}$. Measuring displacements of the considered surface of interest $\mathcal{S}$ consists in minimizing the following weighted functional $\phi^{2}$

$$
\phi^{2}=\sum_{c=1}^{n_{c}} \sum_{j=1}^{n_{s}} \sum_{\mathbf{X} \in \mathcal{S}_{j}} \omega_{j}^{c} \frac{\left(I_{t}^{c}\left(\mathbf{x}^{c}+\mathbf{u}^{c}(\{\mathbf{U}\})\right)-I_{0}^{c}\left(\mathbf{x}^{c}\right)\right)^{2}}{2\left(\sigma^{c}\right)^{2}}
$$

with respect to the unknown degrees of freedom $\{\mathbf{U}\}$, a column vector that gathers the set of elementary degrees of freedom $U_{k}$, for the series of images in the deformed configuration $I_{t}^{c}$ with respect to those in the reference configuration $I_{0}^{c}$. In the present setting, the apparent pixel displacement $\mathbf{u}^{c}$ depends on $\{\mathbf{U}\}$. Let us stress that this formalism allows an arbitrary number 
of cameras (i.e., $n_{c} \geq 2$ ) to be considered, in a similar fashion as standard stereocorrelation (i.e., $n_{c}=2$ ).

The weighting introduced in Equation (3) is associated with the standard deviation $\sigma^{c}$ of acquisition noise, which in the present case is assumed to be white and Gaussian. It is worth noting that this type of formulation allows for different camera definitions in addition to different modalities to be accounted for in a unique formulation $[36,37]$.

Let us consider a perturbation of the 3D displacement field

$$
\delta \mathbf{U}(\mathbf{X})=\sum_{i=1}^{n_{p}} \sum_{j=1}^{3} \delta U_{i j} N_{i}(\mathbf{X}) \mathbf{e}_{j}
$$

For camera $c$, this displacement is projected to

$$
\delta \mathbf{u}^{c}\left(\mathbf{x}^{c}\right)=\left[\boldsymbol{\Pi}^{c}\right] \delta \mathbf{U}(\mathbf{X})
$$

where $\left[\boldsymbol{\Pi}^{c}\right]=\partial \mathbf{x}^{c} / \partial \mathbf{X}$. The latter defines the "kinematic sensitivity" field (no summation over $k$ )

$$
\boldsymbol{\varphi}_{k}^{c}\left(\mathbf{x}^{c}\right)=\delta \mathbf{u}^{c}\left(\mathbf{x}^{c}\right) / \delta U_{k}=\left[\Pi^{c}\right] \mathbf{e}_{k} N_{k}(\mathbf{X})
$$

associated with the degree of freedom $U_{k}$ along direction $\mathbf{e}_{k}$. In turn, this perturbed motion induces a change in the perceived intensity at pixel position $\mathbf{x}^{c}$

$$
\delta I^{c}\left(\mathbf{x}^{c}\right)=\nabla I_{0}^{c}\left(\mathbf{x}^{c}\right) \cdot \delta \mathbf{u}^{c}\left(\mathbf{x}^{c}\right)
$$

that can be rephrased with the "gray level sensitivity" defined as

$$
\psi_{k}^{c}\left(\mathbf{x}^{c}\right)=\delta I^{c}\left(\mathbf{x}^{c}\right) / \delta U_{k}=\nabla I_{0}^{c}\left(\mathbf{x}^{c}\right) \cdot \varphi_{i}^{c}\left(\mathbf{x}^{c}\right)
$$

Equation (8) relates the $3 \mathrm{D}$ motion to its expression in the raw measurement, i.e., the pictures themselves.

This sensitivity analysis allows the inverse problem to be considered, namely, the violation of the conservation of gray levels due to noise, $\eta^{c}\left(x^{c}\right)$, will be 
interpreted as much as possible, in the multiview correlation framework, as due to motion deviation $\delta \mathbf{U}$ with respect to ground truth. This is the origin of displacement uncertainties due to noise. Thus, the presence of noise, $\eta^{c}\left(\mathbf{x}^{c}\right)$, for all camera pixels and all cameras, will lead to the evaluation of a displacement fluctuation $\{\delta \mathbf{U}\}$ given by the minimization of the cost function $\phi^{2}$, or

$$
[\mathcal{H}]\{\delta \mathbf{U}\}=\sum_{c=1}^{n_{c}}\left\{\delta \mathbf{h}^{c}\right\}
$$

where $[\mathcal{H}]=\sum_{c=1}^{n_{c}}\left[\mathbf{H}^{c}\right]$ denotes the global Hessian, and $\left[\mathbf{H}^{c}\right]$ the Hessian associated with each camera $c$

$$
\begin{aligned}
H_{i j}^{c} & =\sum_{k=1}^{n_{s}} \sum_{\boldsymbol{X} \in \mathcal{S}_{k}} \omega_{k}^{c} \frac{\psi_{i}^{c} \psi_{j}^{c}}{2\left(\sigma^{c}\right)^{2}} \\
& =\sum_{k=1}^{n_{s}} \sum_{\boldsymbol{X} \in \mathcal{S}_{k}} \omega_{k}^{c} \frac{\left(\boldsymbol{\nabla} I_{0}^{c} \cdot \boldsymbol{\varphi}_{i}^{c}\right)\left(\boldsymbol{\nabla} I_{0}^{c} \cdot \boldsymbol{\varphi}_{j}^{c}\right)}{2\left(\sigma^{c}\right)^{2}}
\end{aligned}
$$

where the dependence on $\mathbf{x}^{c}$ in the summands has been omitted for the sake of simplicity. The RHS member $\left\{\delta \mathbf{h}^{c}\right\}$ reads

$$
\delta h_{j}^{c}=\sum_{k=1}^{n_{s}} \sum_{\boldsymbol{X} \in \mathcal{S}_{k}} \omega_{k}^{c} \frac{\eta^{c} \psi_{j}^{c}}{2\left(\sigma^{c}\right)^{2}}
$$

Because the mean gray level of Gaussian noise is equal to zero, then the mean displacement fluctuations solely induced by noise vanish, or, in other words, the displacement estimates are unbiased. The covariance matrix of the measured degrees of freedom $\left[\mathbf{C o v}_{\mathbf{u}}\right]=\left\langle\{\delta \mathbf{U}\}\{\delta \mathbf{U}\}^{\top}\right\rangle_{\eta}$ becomes

$$
\left[\operatorname{Cov}_{\mathbf{u}}\right]=[\mathcal{H}]^{-1}
$$

When (iterative) Gauss-Newton schemes are considered to perform the minimization of Equation (3), the global Hessian [H] is computed [38, 1, 39]. Consequently, the covariance matrix $\left[\mathbf{C o v}_{\mathbf{u}}\right]$ is directly known since it simply is the inverse of $[\mathcal{H}]$ in the present setting.

Equation (12), which is the main result of the present paper, corresponds to the generalization of results derived for Q4-DIC [40], T4-DVC [41] and C8DVC [42] to multiview correlation. If local approaches are to be considered, then 
$\mathcal{S}$ corresponds, for instance, to a single surface facet. Conversely, the union of many such facets forming a continuous mesh will lead to global approaches.

Last, a mean-field approximation of the previous result is derived. Long range and short range fluctuations are separated in the expression of the Hessians $\left[\mathbf{H}^{c}\right]$, namely, $\boldsymbol{\nabla} I_{0}^{c}$ is rapidly varying in comparison to $\varphi_{i}^{c}$ and is replaced by its expected value. Therefore, the integral of the tensor $\nabla I_{0}^{c} \otimes \nabla I_{0}^{c}$ is equal to $S_{k}^{c} / 2\left\langle\left\|\nabla I_{0}^{c}\right\|_{2}^{2}\right\rangle \mathbf{I}$, where $S_{k}^{c}$ is the area of the projected support surface of the interpolation function $N_{k}$. Conversely, $[\boldsymbol{\Pi}] \mathbf{e}_{k}$ is very slowly varying for smooth surfaces and can be considered constant over $S_{k}^{c}$ for planar surfaces or surfaces with high curvature radii (and its norm equal to $\Pi_{k}^{c}$ ). Consequently, the square of the interpolation functions are to be integrated to obtain a standard displacement uncertainty for the considered degree of freedom $U_{k}$

$$
\widetilde{\sigma}_{k}^{2} \propto \frac{1}{N_{e}^{2}} \sum_{c=1}^{n_{c}} \frac{4\left(\sigma^{c}\right)^{2}}{\left(\Pi_{k}^{c}\right)^{2}\left\langle\left\|\nabla I_{0}^{c}\right\|_{2}^{2}\right\rangle}
$$

where the coefficient of proportionality will depend on the type of interpolation function.

\section{Analysis of artificial cases}

The aim of this section is to analyze the change of standard displacement uncertainties as functions of mesh sizes, number of evaluation points, surface pattern characteristics, and type of registration analysis. For each analysis, series of 100 pictures are obtained by adding white Gaussian noise to the considered reference picture. Then 100 stereocorrelation analyses are run. From these data, the full covariance matrix can be determined. What is reported in the sequel is only one global quantity per direction. The standard deviation of each kinematic degree of freedom is computed for its 100 evaluations, and its mean level per direction is reported and referred to as standard displacement uncertainty $\sigma_{u}$. 


\section{Test cases}

The following test cases are based upon the NURBS shape that was studied to validate the implementation of a CAD-based stereocorrelation procedure $[18$, 19]. Two different patterns are considered herein:

- The first one corresponds to sprayed paint on a cross-shaped sample made of a vinylester matrix reinforced by E-glass fibers and tested in a biaxial testing machine [43].

- The second one is a classical color painting, converted to gray scale (with a binning of 2 procedure), that underlines the effect of spatial correlations that differ much from a speckle that has been designed specifically for DIC purposes.

These patterns are then expressed in the parametric space of the NURBS patch defining the surface of interest. From this information, they are "drawn" on the 3D surface (Figure 1(a)) thanks to the blending functions of NURBS [44]. With the projection matrices (determined in the first step of the calibration procedure [18]), they are expressed in the camera planes. They finally need to be interpolated at pixel locations. In the present case, a bilinear interpolation scheme was selected. With this procedure, pictures of the reference configuration are created for each considered camera and pattern.

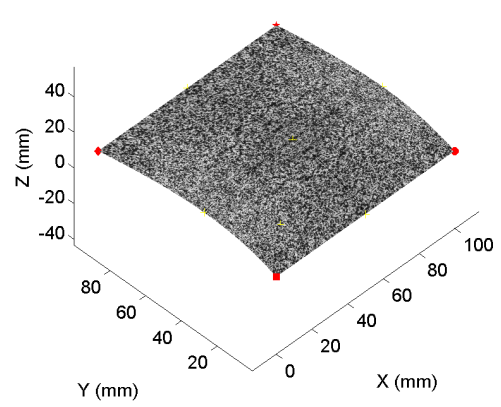

(a)

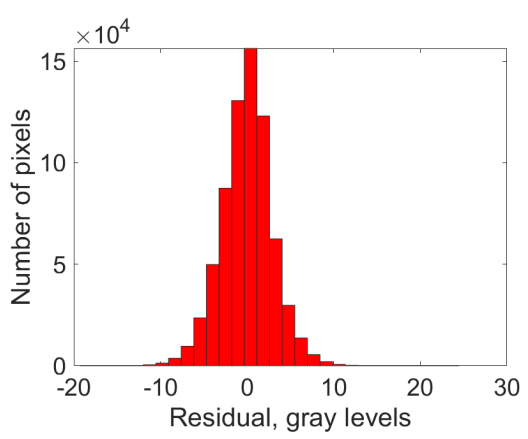

(b)

Figure 1. (a) Random pattern on 3D shape. (b) Gray level histogram of added noise. 
A synthetic Gaussian noise is added to these images before registering them with the previous ones. In order to be representative of the actual experiment, the added noise corresponds to the gray level residuals of the registration between the picture in the reference configuration and that of the first loading step, for which the registration errors can be assumed to be very small. Figure 1(b) shows the histogram of these gray level residuals. As expected from random noise, its mean is close to 0 . Its standard deviation is equal to 2.9 gray levels.

With the selected camera configuration described by the projection matrices, it is possible to get some early indication on the relative displacement uncertainties to be expected due to noise from the sensitivities $\left[\Pi^{c}\right] \mathbf{e}_{k}$. Figure 2 shows the norm of the sensitivity fields for both cameras taken independently, and then combined (i.e., the square root of their squared norms assuming that the noise variance is the same for both cameras) for all three $\mathbf{e}_{k}$ directions. These fields are useful for having a first order estimate of measurement uncertainties (see Equation (13)) when it is assumed that contrasts are uniform in the region of interest and have fluctuations with significantly higher frequencies than those of the sensitivity fields. From this result, it is concluded that a centi-pixel sensitivity is obtained for micrometer displacements in the $X$ and $Y$ directions. Conversely, the $Z$ direction is observed to be about 3.4 times less sensitive. It is expected that the displacement uncertainties in the $X$ and $Y$ directions will be 3.4 times lower than that in the $Z$ direction. Micrometer uncertainties can be expected for $X$ and $Y$ displacements, but the $Z$ direction will be more challenging. 


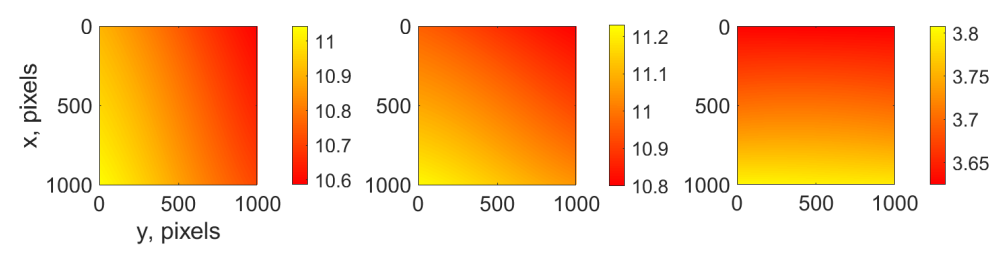

(a) left camera

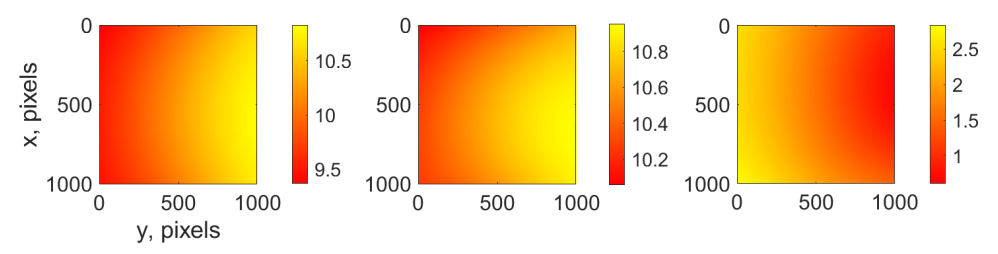

(b) right camera

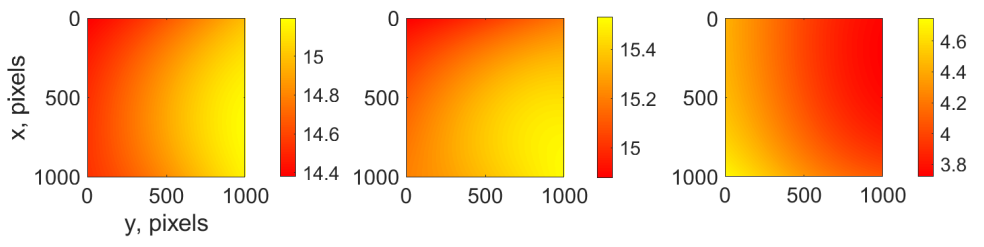

(c) global

Figure 2. Norm of sensitivity fields (expressed in $\mathrm{mpx} / \mu \mathrm{m}$ ) with respect to a motion in $X$ (left), $Y$ (middle), and $Z$ (right) directions. Three cases are shown, namely, the fields for the left (a), right (b) cameras, and their combined effect (c)

In the following, so-called T3-stereocorrelation will be used [21]. It consists in discretizing the surface of interest with a continuous meshes made of 3-noded triangles. In the present case, it is obtained by cutting quadrilaterals in two (Figure 3(a)). The displacements of those vertices (or nodal displacements) are the degrees of freedom to be considered in the correlation procedure. With such a discretization, the measured displacement field is continuous and fully dense 
(i.e., the shape functions of the $\mathrm{T} 3$ elements provide the interpolation to be performed at any location of the region of interest).

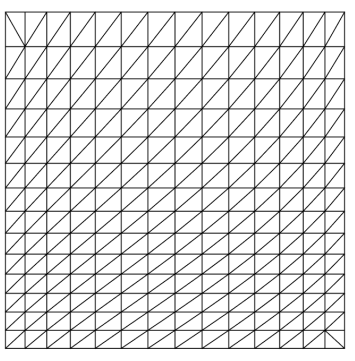

(a)

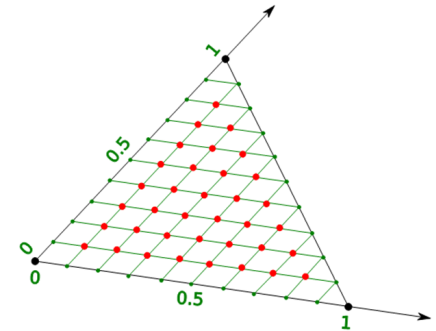

(b)

Figure 3. (a) Example of T3 mesh used in the reported analyses. (b) Depiction of the evaluation points in the parametric space of T3 elements.

In the sequel, meshes consisting of $5 \times 5$ nodes (i.e., 75 degrees of freedom), $10 \times 10$ nodes (i.e., 300 degrees of freedom), $15 \times 15$ nodes (i.e., 675 degrees of freedom, see Figure 3), and $20 \times 20$ nodes (i.e., 1,200 degrees of freedom) will be considered.

With the present setting, there is no master camera and hence pixels are not the key local discretization level. Rather, the 3D surface (or equivalently its parametric space representation) is the master, and all cameras are slaved to it. Conversely, evaluation points are to be defined in the parametric space of each finite element [21], see Figure 3(b). They play the same role as pixels in standard stereocorrelation procedures [1], and as Gauss points in regular finite element analyses [45]. The evaluation points are defined with their barycentric coordinates, $(i /(N+1), j /(N+1),(N+1-i-j) /(N+1))$, where $i$ and $j$ are integers such that $1 \leq i, 1 \leq j$ and $(i+j) \leq(N+1)$. With this setting, $N$ parameterizes the density of evaluation points, and $N(N+1) / 2$ the total number of points in any triangle. It is chosen to define the "equivalent number 
of evaluation points" $N_{e}$ as

$$
N_{e}=\sqrt{\frac{N(N+1)}{2}}
$$

where $N_{e}$ would correspond to the equivalent length of the side of a square having the same number of evaluation points as the triangle. It is worth noting that when the minimum distance between two neighboring evaluation points is less than the pixel scale (in each considered camera [20]) it brings no additional information. Changing the number of evaluation points is equivalent to selecting different scales for the analyses. Such scale changes have to be adjusted in comparison with the correlation length of the random pattern and the scale factor.

\section{Global T3 Stereocorrelation}

In the following analyses, the equivalent number of evaluation points $N_{e}$, as given by Equation (14), ranges from less than 4 to less than 40, which is a very small number. In standard stereocorrelation, it would correspond to square window sizes ranging from 4 to 40 pixels. Consequently, it is expected to be challenging for the correlation procedures. Figure 4 shows the change of the standard displacement uncertainty $\sigma_{u}$ with the equivalent number of evaluation points $N_{e}$ for the finest mesh (i.e., with $20 \times 20$ nodes). Even though the number of evaluation points remains small, micrometer to sub-micrometer uncertainties are observed for the $X$ and $Y$ directions. The uncertainty in the $Z$ direction is four times higher, which confirms the a priori analyses performed by studying the sensitivity fields (Figure 2). Further, the displacement uncertainty is inversely proportional to $N_{e}$, which is to be expected for registrations using 2D pictures [40]. This first result exemplifies the trade-off between displacement uncertainties and the number of evaluation points (for global stereocorrelation). 


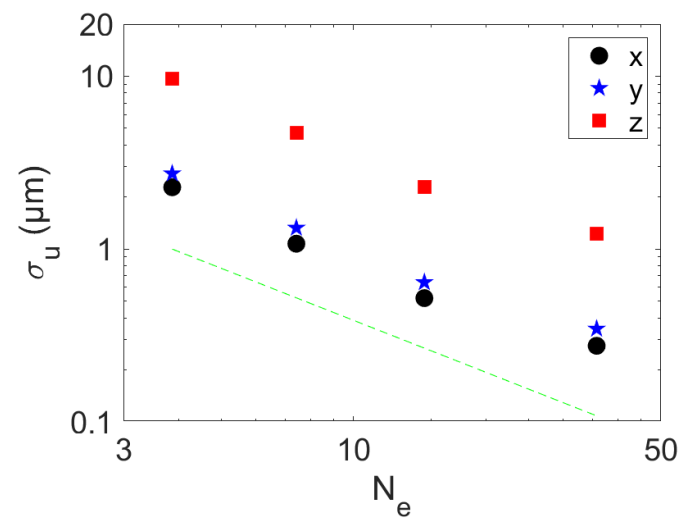

Figure 4. Standard displacement uncertainty $\sigma_{u}$ vs. equivalent number of evaluation points $N_{e}$ for the mesh made of $20 \times 20$ nodes (global analyses). The dashed line depicts the power-law dependence with an exponent -1 .

The effect of the number of nodes is now investigated with the same series of evaluation points as before within each triangular element. Figure 5 shows that there is virtually no effect of the discretization level because the sought displacement field being a constant (uniformly equal to zero) no discretization error is induced by a change in mesh fineness. It is worth remembering that the mesh defines the discretization of the kinematics. The important property is that the evaluation points are assumed to be sufficiently dilute as compared to the texture correlation length and the distance over which gradients are computed so that there is no dependence. For the present sensitivity analysis to acquisition noise, the key parameter is the equivalent number of evaluation points, which defines the number of data used to evaluate the kinematic degrees of freedom. 


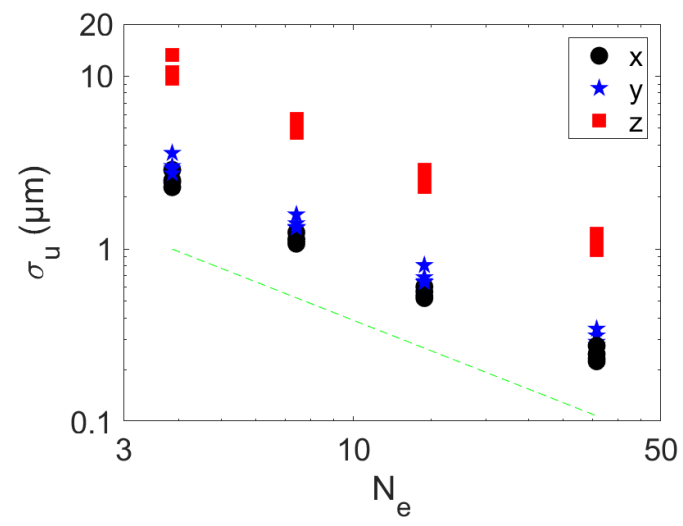

(a)

Figure 5. Standard displacement uncertainty $\sigma_{u}$ vs. equivalent number of evaluation points for four different meshes (global analyses). The dashed line depicts the power-law dependence with an exponent -1 .

The previous results should now be compared with the a priori estimates based upon the inverse of the global Hessian of the multiview system. However, the inversion of the Hessian is a costly operation. A very simple approximation consists in retaining only the diagonal of the Hessian, $\mathcal{H}_{i j}^{d i a}=\mathcal{H}_{i j} \delta_{i j}$. This inverse of the latter is trivially computed and offers a very good approximation as will be demonstrated at the end of this paper. Figure 6 shows that there is a very good correlation, for all components of the displacement fields between the a priori estimate based on $\mathcal{H}^{d i a}$ and the a posteriori results. When a linear interpolation is sought, the slope is equal to 0.98 , which is very close to unity. 


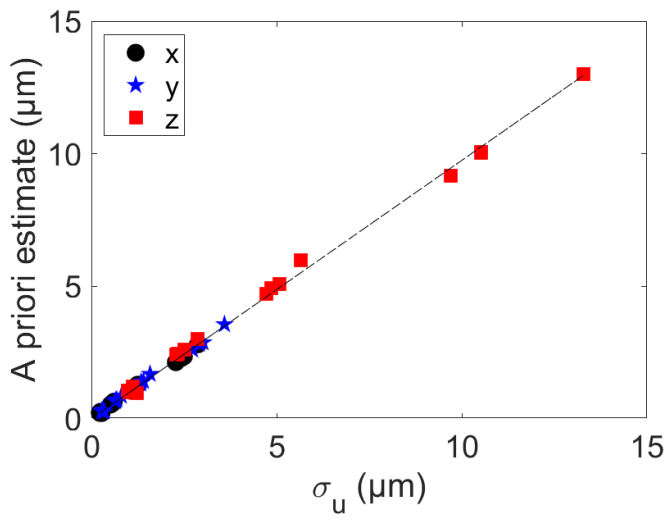

Figure 6. Comparison of a priori and a posteriori standard displacement uncertainties $\sigma_{u}$ for all global analyses. The dashed line depicts a linear interpolation with a slope of 0.98 .

\section{Local T3 stereocorrelation}

The preceding analyses will be repeated for local registrations and compared with global results. It is worth noting that the current setting is different from regular stereocorrelation techniques [1]. First, T3 elements will still be considered as opposed to quadrilateral interrogation windows. Second, the registration is not performed as a master/slave setting of the cameras but in terms of the degrees of freedom defined in the 3D space. Third, as already mentioned, the present implementation considers evaluation points in the parametric space of the elements, which then translate into (non-integer) pixel locations thanks to the projection matrices. Fourth, contrary to standard practice, the uncertainties of nodal displacements are still considered and not the mean displacement. Last, the actual implementation consists in disconnecting each element from all others so that a local analysis is performed independently. With the present choice, there is no element overlap so that both approaches are compared with the same set of elements. 
In the following analyses, the range of evaluation points $N$ was increased from 20 to 400 so that the equivalent number $N_{e}$ ranged from 15 to 300 . This choice will become clearer in the sequel. It suffices to mention here that the spatial resolutions of local and global approaches are different [40]. Figure 7 shows the change of the standard displacement uncertainties as functions of the equivalent number of evaluation points. All the general trends observed in global analyses also apply to local approaches (i.e., power -1 decrease with $N_{e}$, quasi independence with the mesh, factor 3.5 ratio between $X$ and $Y$ components in comparison with the $Z$ direction.

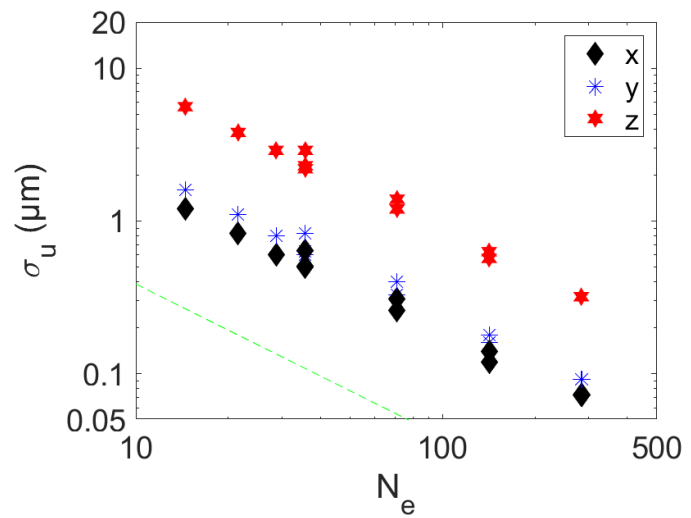

Figure 7. Standard displacement uncertainty $\sigma_{u}$ vs. equivalent number of evaluation points $N_{e}$ for four different tessellations (local analyses). The dashed line depicts the power-law dependence with an exponent -1 .

\section{Comparisons}

In order to compare both sets of results in a more quantitative way, all of them are gathered in Figure 8. There is a clear offset by a factor of about 2.5 between the uncertainties of local and global approaches. This effect can be understood by the spatial resolution associated with both approaches, which 
was already discussed for 2D-DIC in which local and global Q4 approaches were compared [40].

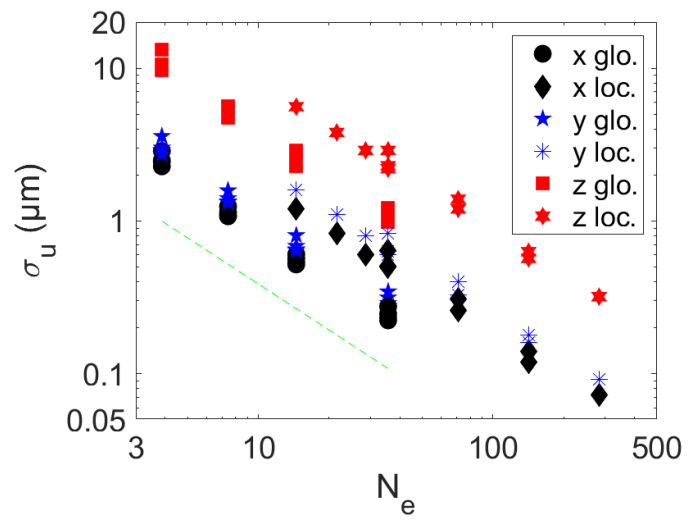

Figure 8. Standard displacement uncertainty $\sigma_{u}$ vs. equivalent number of evaluation points $N_{e}$ for four different meshes and different registration procedures. The dashed line depicts the power-law dependence with an exponent -1 .

The spatial resolution, namely, the number of equivalent evaluation points that are used to measure a given degree of freedom is assessed for both approaches with the same node distribution. For local approaches (superscript l), the spatial resolution is simply the equivalent number of evaluation points $N_{e}^{l}$ since each T3 element is independently analyzed. For global analyses, (superscript $g$ ), the situation is a bit more complex [40], see Figure 9(a). For corner nodes, in the present case, two elements are considered. Consequently, the spatial resolution is equal to $\sqrt{2} N_{e}^{l}$. For edge nodes, three elements are mostly used (i.e., the spatial resolution is equal to $\sqrt{3} N_{e}^{l}$ ). Last, for inner nodes the nodal connectivity is $\operatorname{six}\left(i . e\right.$. , the spatial resolution is equal to $\sqrt{6} N_{e}^{l}$ ). It follows that an upper bound to the spatial resolution $N_{e}^{g}$ for global T3 multiview correlation is

$$
N_{e}^{g} \leq \sqrt{6} N_{e}^{l}
$$


which is asymptotically reached when the number of edge nodes becomes negligible as compared to inner nodes $\left(N_{e} \gg 1\right)$. This result is illustrated in Figure 9(b) for the different discretizations analyzed herein. It is observed that for the finest mesh the upper bound is a very good approximation. Conversely for coarse meshes, the relative weight of corner and edge nodes being more important, the actual spatial resolution departs more significantly from its upper bound.

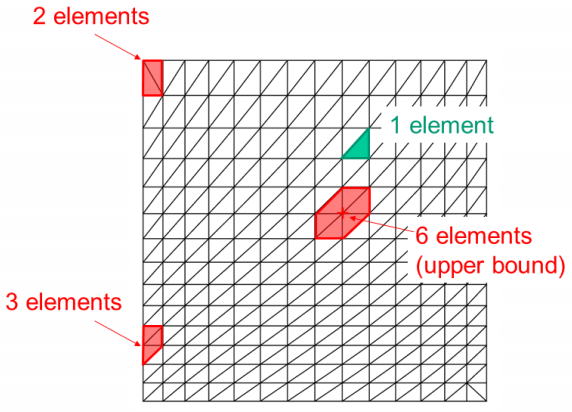

(a)

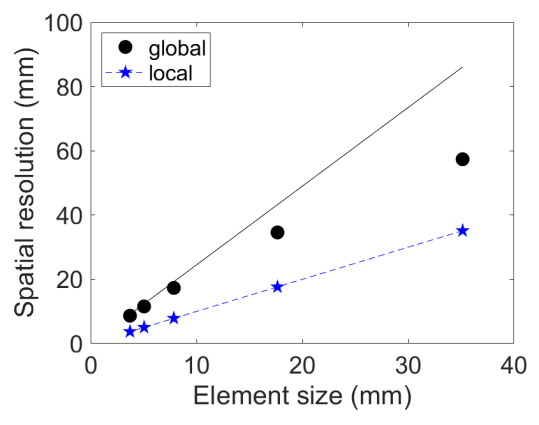

(b)

Figure 9. Spatial resolutions for local and global T3 multiview correlations. (a) Depiction of the number of elements used in local and global analyses for the evaluation of nodal displacements. (b) Spatial resolutions for the meshes studied herein for global and local analyses.

The results of Figure 8 are now reported in Figure 10 using the spatial resolution discussed before. There is a very good correlation for both series of analyses. It is found that the standard displacement resolution is inversely proportional to the spatial resolution, as for 2D-DIC analyses [40]. The factor 2.5 , which was anticipated from the raw results of Figure 8 , is now understood as the $\sqrt{6}$ ratio in spatial resolution. 


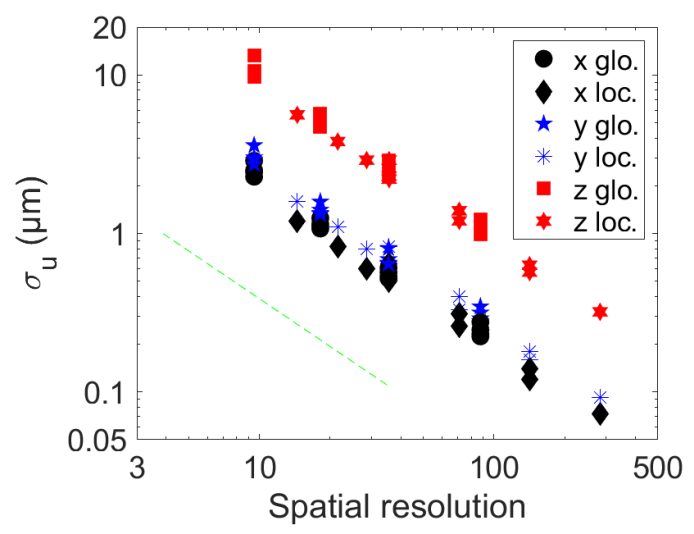

Figure 10. Standard displacement uncertainty $\sigma_{u}$ vs. spatial resolution $\left(N_{e}^{g}\right.$ or $\left.N_{e}^{l}\right)$ for different meshes, tessellations and registration procedures. The dashed line depicts the power-law dependence with an exponent -1 .

This comparison can be rationalized in two different ways. If the meshes are identical for local and global analyses, the fact that each T3 element has a nodal connectivity that can be as high as 6 will lead to a decrease of standard displacement uncertainties by a factor at most equal to $1 / \sqrt{6}$. Conversely, in order to achieve the same displacement uncertainties, less (i.e., $1 / \sqrt{6}$ ) evaluation points need to be considered in global approaches than in local analyses. Since the connectivities of edge and corner nodes are lower than those of inner nodes, special attention should be exercised if a uniform uncertainty is sought (i.e., adapting the mesh as was proposed for Q4-DIC [40]).

\section{Painting}

The final analysis consists in using the picture of an actual paint, namely, the Descent of the Cross (Figure 11(a)) that was recently restored and attributed to Sebastian Düring [46]. During its restoration, NURBS-based stereocorrelation was employed to analyze two tensioning systems of the canvas in order to minimize wrinkling [47]. As stated above, the picture was converted to gray 
levels for the correlation analysis. No pattern was deposited and even though the contrast is rather low in comparison with speckle patterns (Figure 1), it was shown that very fine analyses could be preformed thanks to global NURBSbased analyses. It is therefore an interesting test case to probe both approaches.

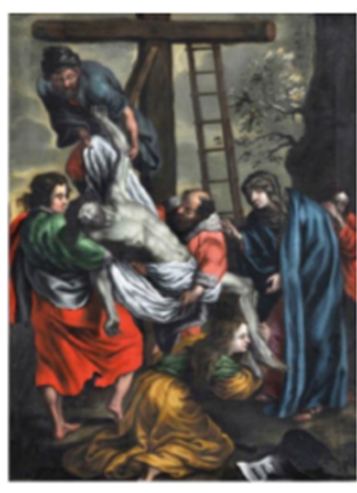

(a)

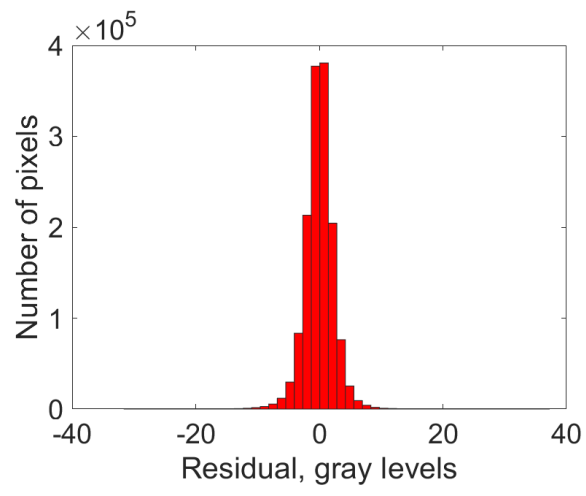

(b)

Figure 11. (a) Restored Descent of the Cross [46]. (b) Gray level histogram of added noise.

Figure 11(b) shows the gray level histogram associated with acquisition noise. The mean is very close to 0 and the standard deviation is equal to 2.3 gray levels. As in the previous case, it will be used to create noisy images to be registered with their noise-free references. Given the fact that this pattern is deemed very difficult, a larger number of evaluation points was considered, namely, from 7 to 64 . Figure 12 shows the change of the standard displacement uncertainties with $N_{e}$ for the four considered meshes. As compared to the previous results, the same general trends are observed (i.e., uncertainty in $Z$ direction about four times higher than those along directions $X$ and $Y$, inverse proportionality with equivalent number of evaluation points, mesh insensitivity). 


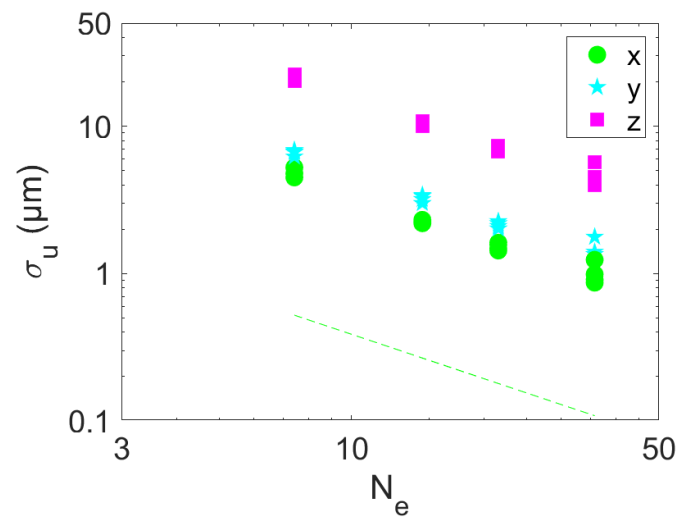

Figure 12. Standard displacement uncertainties $\sigma_{u}$ vs. equivalent number of evaluation points $N_{e}$ for four different meshes (global analyses). The dashed line depicts the power-law dependence with an exponent -1 .

When compared with the a priori estimates of displacement uncertainties based on the (diagonal approximation of) Hessian of the registration procedure, it is observed in Figure 13 that the correlation is again very satisfactory with a slope of 1.02. An offset is observed for the $X$-direction, whose origin has not been elucidated.

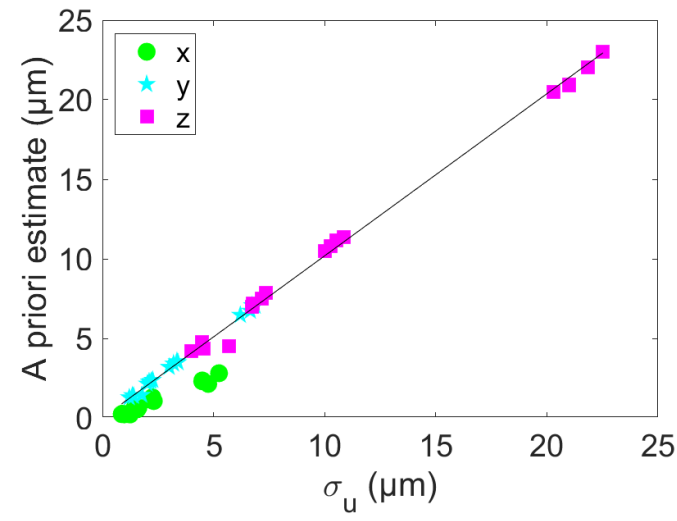

Figure 13. Comparison of a priori and a posteriori standard displacement uncertainties $\sigma_{u}$ for all analyzed cases. The solid line depicts a linear interpolation with a slope of 1.02 . 
Moreover, the displacement uncertainties are significantly higher than in the previous case (with the global approach). In quantitative terms, there is a fourfold increase of all uncertainties when compared with the random pattern (Figure 14). This effect is related to the acquisition noise (whose variance is very close for the pictures analyzed herein) and the contrast of the pictures. The RMS contrast (i.e., average of norm half of the picture gradient over the region of interest) of the speckle pattern is equal to 20.6 gray level per pixel, and 4.8 for the Descent of the Cross. The same order of magnitude is observed.

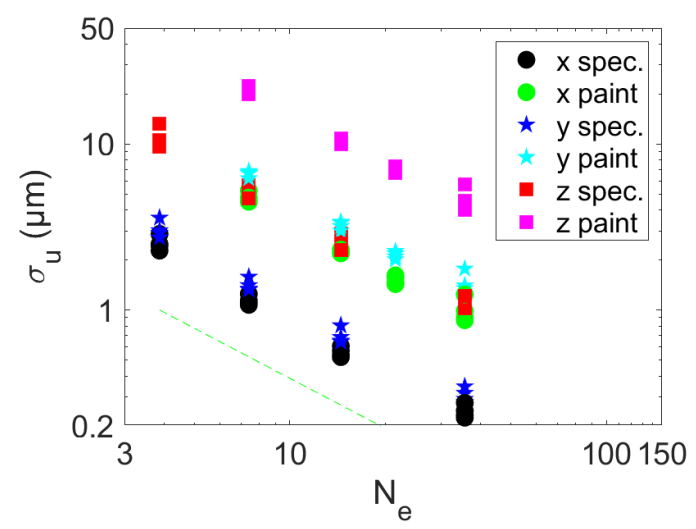

Figure 14. Standard displacement uncertainty $\sigma_{u}$ vs. equivalent number of evaluation points $N_{e}$ for four different meshes and two different patterns. The dashed line depicts the power-law dependence with an exponent -1 .

To account for all the different conditions illustrated in Figure 14, namely, contrast, in-plane and out-of "plane components," number of evaluation points, and acquisition noise, the actual standard displacement uncertainties are compared to their mean-field estimates using an extension of Equation (13)

$$
\widetilde{\sigma}_{u}^{2}=\frac{1}{N_{e}^{2}} \sum_{c=1}^{n_{c}} \frac{4\left(\sigma^{c}\right)^{2}}{\left(\Pi_{u}^{c}\right)^{2}\left\langle\left\|\nabla I_{0}^{c}\right\|_{2}^{2}\right\rangle}
$$

where $\Pi_{u}^{c}$ denotes the mean (over the whole ROI) pixel/m sensitivity for the studied component of displacement, which is evaluated from $\left[\Pi^{c}\right]$. If the previous 
mean-field result is consistent with the numerical results, the ratio of $\sigma_{u}$ by its approximation $\widetilde{\sigma}_{u}$ should collapse onto a single horizontal line $\Sigma_{u}$. Figure 15 shows that this trend is observed for the two analyzed cases with $\Sigma_{u}=1.2 \pm 0.3$. In particular, the normalized uncertainties no longer depend on the equivalent number of evaluation points, nor on the "speckle" patterns.

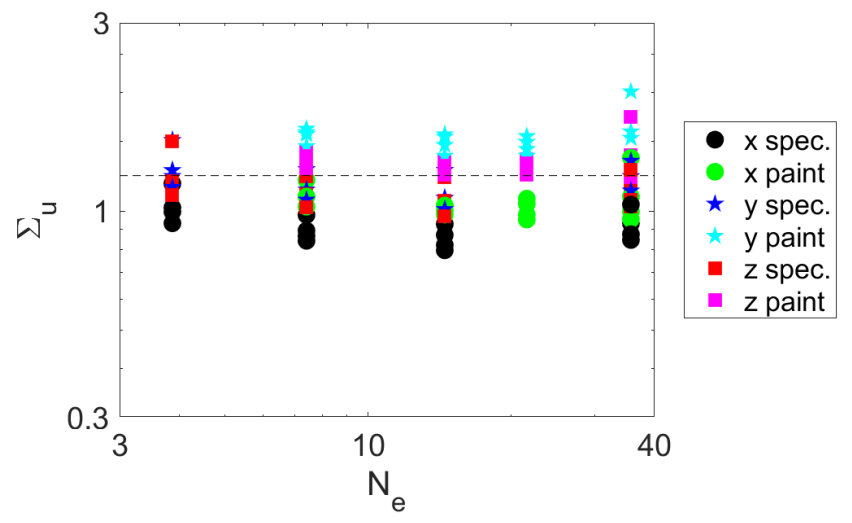

Figure 15. Dimensionless standard displacement uncertainty $\Sigma_{u}=\sigma_{u} / \widetilde{\sigma}_{u}$ vs. equivalent number of evaluation points $N_{e}$ for four different meshes and two different patterns. The dashed line depicts the mean level (i.e., $1.2 \pm 0.3$ ).

\section{Conclusion}

The displacement uncertainties have been evaluated within the framework of multiview correlation. The latter enables multiple (i.e., more than two) cameras with various modalities to be considered in a unique registration step. In the present case, the surface of interest was parameterized with triangular tessellations or meshes made of triangular elements. Such hypotheses lead to local and global T3 stereocorrelation when only two cameras with the same modality are considered [21, 22].

A closed-form expression of the covariance matrix of the measured degrees of freedom was derived when only acquisition noise was considered (i.e., noise floor 
level [32]). With the present setting, it corresponds to the Hessian used in the minimization scheme of the registration algorithm. Such results allow for a priori estimates of measurement uncertainties. They were probed on two test cases for which the speckle pattern was significantly different (i.e., there was a fourfold difference in terms of contrast). Very consistent agreements were observed when the a priori estimates were compared with stereocorrelation measurements on series of 100 pictures in which experimental noise was added. A unique collapse for standard displacement uncertainties ranging over more than two orders of magnitude was achieved by accounting for the number of evaluation points, the mean contrast, and the average sensitivity for the three components of displacements.

Uncertainty quantifications associated with local and global registrations were also compared. The main difference, as already observed in 2D-DIC [40], and DVC $[42,41]$ was due to the spatial resolution, which in the present case was a ratio of $\sqrt{6}$, and that led to lower uncertainties (by the same factor), for the same distribution of evaluation points, for the global approach in comparison to the local registrations.

The cases studied herein were only based on perturbing reference images with experimentally observed noise. Similar analyses should now be performed for real experiments. They should also account for rigid body motions to fully characterize the performances of the correlation code [35]. Additional evaluations would also be needed to assess the uncertainties associated with the calibration step, and the shape correction step by extending the framework utilized herein. Additional biases such as those induced by optical distortions may also be accounted for. Last, let us note that the present tools are very useful when designing and optimizing experiments monitored by multiview systems [48]. 


\section{Acknowledgments}

This work was partly supported within PRC MECACOMP, a French research project co-funded by DGAC and SAFRAN Group, managed by SAFRAN Group and involving SAFRAN Group, ONERA and CNRS.

\section{Appendix: Evaluation of the standard deviation $\sigma^{c}$}

The following appendix discusses the way of assessing the standard deviation $\sigma^{c}$ of the noise level for any evaluation point $\mathbf{x}^{c}$. It is assumed that the noise $\eta^{c}$ at pixel locations $\mathbf{x}^{c}$ has been characterized. The evaluation of the gray levels at position $\mathbf{x}^{c}$ requires the use of an interpolation scheme. Let the latter be defined with a set of $n_{i}$ interpolation functions $N_{i}$ such that

$$
I^{c}\left(\mathbf{x}^{c}\right)=\sum_{i=1}^{n_{i}} I^{c}\left(\mathbf{x}_{i}^{c}\right) N_{i}\left(\mathbf{x}^{c}\right)
$$

where $\mathbf{x}_{i}^{c}$ denote pixel positions in the considered image $I^{c}$ acquired by camera $c$. In the following, it is assumed that acquisition noise $\eta^{c}$ is Gaussian (with zero mean) and characterized by the covariance matrix $\left[\mathbf{C o v}_{\eta^{c}}\right]$, which is positive semi-definite. Given the fact that the interpolation scheme is linear with respect to gray levels $I^{c}\left(\mathbf{x}_{i}^{c}\right)$ (Equation (17)), the noise propagation is straightforward, and the variance $\left(\sigma^{c}\right)^{2}$ for the considered evaluation point $\mathbf{x}^{c}$ reads

$$
\left(\sigma^{c}\right)^{2}\left(\mathbf{x}^{c}\right)=\left\|\left\{\mathbf{N}\left(\mathbf{x}^{c}\right)\right\}\right\|_{\left[\mathbf{C o v}_{\eta^{c}}\right]}^{2}
$$

where $\|\cdot\|_{\left[\mathbf{C o v}_{\eta^{c}}\right]}$ denotes the norm constructed with the covariance matrix $\left[\mathbf{C o v}_{\eta^{c}}\right]$, and $\left\{\mathbf{N}\left(\mathbf{x}^{c}\right)\right\}$ the column vector gathering the interpolation functions $N_{i}$ evaluated at the position $\mathbf{x}^{c}$. When noise is white with identical variance $\sigma_{\eta^{c}}^{2}$ (i.e., the covariance matrix reduces to $\sigma_{\eta^{c}}^{2}[\mathbf{I}]$, where $[\mathbf{I}]$ is the identity matrix), the previous result becomes

$$
\left(\sigma^{c}\right)^{2}\left(\mathbf{x}^{c}\right)=\sigma_{\eta^{c}}^{2}\|\{\mathbf{N}\}\|_{2}^{2}
$$


where $\|\cdot\|_{2}$ is the standard L2-norm.

In the present analyses, bilinear interpolations were considered. Consequently, four interpolation functions were needed

$$
N_{i}\left(x^{c}, y^{c}\right)=\sigma_{\eta^{c}}^{2}\left(\frac{1}{2} \pm x^{c}\right)\left(\frac{1}{2} \pm y^{c}\right)
$$

for $-1 / 2 \leq x^{c}, y^{c} \leq 1 / 2$ (i.e., the pixel centers are located at half integers). The variance $\sigma^{2}$ then reads

$$
\begin{aligned}
\left(\sigma^{c}\right)^{2}\left(x^{c}, y^{c}\right) & =\sigma_{\eta^{c}}^{2} \sum\left(\frac{1}{2} \pm x^{c}\right)^{2}\left(\frac{1}{2} \pm y^{c}\right)^{2} \\
& =\sigma_{\eta^{c}}^{2}\left(\frac{1}{4}+\left(x^{c}\right)^{2}+\left(y^{c}\right)^{2}+4\left(x^{c}\right)^{2}\left(y^{c}\right)^{2}\right)
\end{aligned}
$$

If a uniform sampling is assumed (i.e., a large number number of evaluation points), the fluctuations of $\left(\sigma^{c}\right)^{2}$ wrt. $x^{c}$ and $y^{c}$ are neglected and approximated by the average value with a uniform distribution

$$
\left(\sigma^{c}\right)^{2} \approx \int_{-1 / 2}^{1 / 2} \int_{-1 / 2}^{1 / 2}\left(\sigma^{c}\right)^{2}(x, y) \mathrm{d} x \mathrm{~d} y=\frac{4}{9} \sigma_{\eta^{c}}^{2}
$$

or equivalently,

$$
\sigma^{c} \approx \frac{2}{3} \sigma_{\eta^{c}}
$$

It is worth remembering that when the evaluation points are separated enough (i.e., neighboring evaluation points would not have any correlation, as in the present case) there is no need to compute the covariance matrix, which would only have diagonal terms, and the present setting (see Equation (3)) is still optimal.

\section{References}

[1] Sutton M, Orteu J and Schreier H. Image correlation for shape, motion and deformation measurements: Basic Concepts, Theory and Applications. New York, NY (USA): Springer, 2009. 
[2] Sutton M. Computer vision-based, noncontacting deformation measurements in mechanics: A generational transformation. Applied Mechanics Reviews 2013; 65(AMR-13-1009): 050802.

[3] GOM. ARAMIS. Sensors for high-precision motion and deformation analyses. 2019 (accessed). URL www.gom.com/metrology-systems/ aramis.html.

[4] Holo3. CorreliSTC. Link between Experiments and Simulations. 2019 (accessed). URL www.correli-stc.com/.

[5] Eikosim. EikoTwin DIC - Global field measurement. 2019 (accessed). URL eikosim.com/en/eikotwin-2/.

[6] Dantec Dynamics. Q-400 DIC - Digital Image Correlation System Measurement of Shape, Displacement and Strain. 2019 (accessed). URL www . dantecdynamics.com/q-400-dic/.

[7] La Vision. StrainMaster. 2019 (accessed). URL www.lavision.de/en/ products/strainmaster/.

[8] Correlated Solutions. VIC-3D. Non-Contact Full-Field 3D Strain Measurement System. 2019 (accessed). URL www.correlatedsolutions. com/vic-3d/.

[9] Luo P, Chao Y, Sutton M et al. Accurate measurement of threedimensional deformations in deformable and rigid bodies using computer vision. Experimental Mechanics 1993; 33: 123-132.

[10] Luo P, Chao Y and Sutton M. Application of stereo vision to three-dimensional deformation analyses in fracture experiments. Optical Engineering 1994; 33(3): 981-990. 
[11] Helm J, McNeill S and Sutton M. Improved three-dimensional image correlation for surface displacement measurement. Optical Engineering 1996; 35(7): 1911-1920.

[12] Orteu JJ, Garric V and Devy M. Camera calibration for 3D reconstruction: application to the measurement of $3 \mathrm{D}$ deformations on sheet metal parts. In Refregier P and Ahlers RJ (eds.) New Image Processing Techniques and Applications: Algorithms, Methods, and Components II, volume 3101. International Society for Optics and Photonics, SPIE, pp. 252-263.

[13] Lavest JM, Viala M and Dhome M. Do we really need an accurate calibration pattern to achieve a reliable camera calibration? In Burkhardt H and Neumann B (eds.) Computer Vision - ECCV'98. Berlin, Heidelberg: Springer Berlin Heidelberg, pp. 158-174.

[14] Besl $\mathrm{P}$ and McKay N. Method for registration of 3-d shapes. In Sensor Fusion IV: Control Paradigms and Data Structures, volume 1611. International Society for Optics and Photonics, pp. 586-607.

[15] Zhu L, Barhak J, Srivatsan V et al. Efficient registration for precision inspection of free-form surfaces. The International Journal of Advanced Manufacturing Technology 2007; 32(5-6): 505-515.

[16] Senin N, Colosimo B and Pacella M. Point set augmentation through fitting for enhanced icp registration of point clouds in multisensor coordinate metrology. Robotics and Computer-Integrated Manufacturing 2013; 29(1): $39-52$.

[17] F Hild and S Roux. Method of three-dimensional stereo-correlation measurements using a parametric representation of the measurement object. Patent family FR2991448B1, EP2856424B1, US9733071B2, 
ES2700975T3, 2012. URL patents.google.com/patent/FR2991448B1/ en.

[18] Beaubier B, Dufour J, Hild F et al. CAD-based calibration of a 3D-DIC system: Principle and application on test and industrial parts. Experimental Mechanics 2014; 54(3): 329-341.

[19] Dufour JE, Beaubier B, Hild F et al. CAD-based displacement measurements. Principle and first validations. Experimental Mechanics 2015; 55(9): 1657-1668.

[20] Dufour JE, Hild F and Roux S. Shape, Displacement and Mechanical Properties from Isogeometric Multiview Stereocorrelation. Journal of Strain Analysis for Engineering Design 2015; 50(7): 470-487.

[21] Dubreuil L, Dufour JE, Quinsat Y et al. Mesh-based shape measurements with stereocorrelation. Experimental Mechanics 2016; 56(7): 1231-1242.

[22] Pierré JE, Passieux JC and Périé JN. Finite Element Stereo Digital Image Correlation: Framework and Mechanical Regularization. Experimental Mechanics 2017; 57(3): 443-456.

[23] Dufour J, Beaubier B, Roux S et al. Displacement measurement using CAD-based stereo-correlation with meshes. In ICEM conference.

[24] Rao C. Information and the accuracy attainable in the estimation of statistical parameters. Bulletin of the Calcutta Mathematical Society 1945; 37: 81-89.

[25] Cramér H. Mathematical Methods of Statistics. Princeton, NJ (USA): Princeton Univ. Press, 1946.

[26] Wang YQ, Sutton MA, Ke XD et al. On Error Assessment in Stereo-based Deformation Measurements. Experimental Mechanics 2011; 51(4): 405-422. 
[27] Ke XD, Schreier HW, Sutton MA et al. Error Assessment in Stereo-based Deformation Measurements. Experimental Mechanics 2011; 51(4): 423-441.

[28] Reu P. A study of the influence of calibration uncertainty on the global uncertainty for digital image correlation using a monte carlo approach. Experimental Mechanics 2013; 53(9): 1661-1680.

[29] Zhu C, Yu S, Liu C et al. Error estimation of 3D reconstruction in 3D digital image correlation. Measurement Science and Technology 2019; 30(2): 025204 .

[30] Balcaen R, Wittevrongel L, Reu PL et al. Stereo-DIC Calibration and Speckle Image Generator Based on FE Formulations. Experimental Mechanics 2017; 57(5): 703-718.

[31] Balcaen R, Reu P, Lava P et al. Stereo-dic uncertainty quantification based on simulated images. Experimental Mechanics 2017; 57(6): 939-951.

[32] Standardization, Good Practices, and Uncertainty Qunatification Committee. A Good Practices Guide for Digital Image Correlation. International Digital Image Correlation Society (iDICs).

[33] Fréchet M. Sur l'extension de certaines evaluations statistiques au cas de petits échantillons. Revue de l'Institut International de Statistique / Review of the International Statistical Institute 1943; 11(3/4): 182-205.

[34] Darmois G. Sur les limites de la dispersion de certaines estimations. Revue de l'Institut International de Statistique / Review of the International Statistical Institute 1945; 13(1/4): 9-15.

[35] ASD-STAN prEN 4861 P1. Metrological assessment procedure for kinematic fields measured by digital image correlation. 2018. URL /www.asd-stan.org/downloads/asd-stan-pren-4861-p1. 
[36] Charbal A, Dufour JE, Hild F et al. Hybrid stereocorrelation using infrared and visible light cameras. Experimental Mechanics 2016; 56: 845-860.

[37] Wang Y, Charbal A, Dufour JE et al. Hybrid multiview correlation for measuring and monitoring thermomechanical fatigue test. Experimental Mechanics 2019; DOI:10.1007/s11340-019-00500-8.

[38] Lucas B and Kanade T. An iterative image registration technique with an application to stereo vision. In 7th International Joint Conference on Artificial Intelligence. pp. 674-679.

[39] Hild F and Roux S. Digital image correlation. In Rastogi P and Hack E (eds.) Optical Methods for Solid Mechanics. A Full-Field Approach. Weinheim (Germany): Wiley-VCH, pp. 183-228.

[40] Hild F and Roux S. Comparison of local and global approaches to digital image correlation. Experimental Mechanics 2012; 52(9): 1503-1519.

[41] Hild F, Bouterf A, Chamoin L et al. Toward 4d mechanical correlation. Advanced Modeling and Simulation in Engineering Sciences 2016; 3(1): 126.

[42] Leclerc H, Périé J, Hild F et al. Digital volume correlation: What are the limits to the spatial resolution? Mechanics ${ }^{6}$ Industry 2012; 13: 361-371.

[43] Claire D, Hild F and Roux S. Identification of damage fields using kinematic measurements. Comptes Rendus Mécanique 2002; 330: 729-734.

[44] Piegl L and Tiller W. The NURBS Book - 2nd Edition. Springer, 1997.

[45] Zienkievicz O and Taylor R. The Finite Element Method. 4th edition, London (UK): McGraw-Hill, 1989. 
[46] Grau S. Conservation et restauration de la Descente de croix conservée dans le chœur de la basilique de Saint-Maurice en Valais. Technical report, Ecole de Condé, Paris, 2014.

[47] Dufour JE, Gonnet E, Grau S et al. On the analysis of canvas wrinkling via isogeometric stereocorrelation. International Journal of Solids and Structures 2018; 154: 114-123.

[48] Vitse M, Poncelet M, Iskef A et al. Toward virtual design and optimization of a structural test monitored by a multi-view system. Journal of Strain Analysis for Engineering Design 2020; DOI:10.1177/0309324720910887. 